\title{
Some limit properties for a hidden inhomogeneous Markov chain
}

\author{
Yun Dong ${ }^{1 *}$ (D), Fang-qing Ding ${ }^{2}$ and Qi-feng Yao ${ }^{3}$
}

"Correspondence: 405388629@qq.com

${ }^{1}$ School of Mathematics, Maanshan Teachers' College, Maanshan, China Full list of author information is available at the end of the article

\begin{abstract}
This paper presents a general strong limit theorem for delayed sum of functions of random variables for a hidden time inhomogeneous Markov chain (HTIMC), and as corollaries, some strong laws of large numbers for HTIMC are established thereby.
\end{abstract}

MSC: $60 \mathrm{~F} 15 ; 94 \mathrm{~A} 17$

Keywords: Inhomogeneous hidden Markov chain; Delayed sum; Law of large numbers

\section{Introduction}

Hidden Markov chain is an important branch of Markov chain theory. A classical hidden Markov model was first introduced by Baum and Petrie [1]. It provides a flexible model that is very useful in different areas of applied probability and statistics. Examples are found in machine recognition, like speech and optical character recognition, and bioinformatics. The power of these models is that they can be very efficiently implemented and simulated. In recent years, many new theories were introduced into hidden time inhomogeneous Markov chain (HTIMC) theory. G.Q. Yang et al. [2] gave a law of large numbers for countable hidden time inhomogeneous Markov models. In addition, delayed sums of random variables were first discussed by Zygmund [3]. Gut and Stradtmüller [4] studied the strong law of large numbers for delayed sums of random fields. Wang and Yang [5] studied the generalized entropy ergodic theorem with a.e. and $\mathcal{L}_{1}$ convergence for time inhomogeneous Markov chains. Wang [6,7] discussed the limit theorems of delayed sums for row-wise conditionally independent stochastic arrays and a class of asymptotic properties of moving averages for Markov chains in Markovian environments.

In the classical studies there are two simplest models for predicting: the mean model and the random walk model [8]. These two models use all the historical information. But we often encounter time series that appear to be "locally stationary", so we can take an average of what has happened in some window of the recent past. Based on this idea and the above researches, the main focus of this paper is to obtain a general strong limit theorem of delayed sums of functions of random variables for an HTIMC, and as corollaries, some strong laws of large numbers for HTIMC are established thereby.

The remainder of this paper is organized as follows: Sect. 2 gives a brief description of the HTIMC and related lemmas. Section 3 presents the main results and the proofs.

(c) The Author(s) 2018. This article is distributed under the terms of the Creative Commons Attribution 4.0 International License (http://creativecommons.org/licenses/by/4.0/), which permits unrestricted use, distribution, and reproduction in any medium, provided you give appropriate credit to the original author(s) and the source, provide a link to the Creative Commons license, and indicate if changes were made. 


\section{Preliminaries}

In this section we list some fundamental definitions and related results that are needed in the next section.

Let $(\Omega, \mathcal{F}, \mathbb{P})$ be the underling probability space and $\zeta=(\xi, \eta)$ a random vector, where $\xi=\left(\xi_{0}, \xi_{1}, \ldots\right)$ and $\eta=\left(\eta_{0}, \eta_{1}, \ldots\right)$ are two different stochastic processes, $\eta$ is hidden ( $\eta$ takes values in set $\left.\mathcal{Y}=\left\{\omega_{0}, \omega_{1}, \ldots, \omega_{b}\right\}\right)$ and $\xi$ is observable ( $\xi$ takes values in set $\left.\mathcal{X}=\left\{\theta_{0}, \theta_{1}, \ldots, \theta_{d}\right\}\right)$.

We first recall the definition of a hidden time inhomogeneous Markov chain (HTIMC) $\zeta=(\xi, \eta)=\left\{\xi_{n}, \eta_{n}\right\}_{n=0}^{\infty}$ with hidden chain $\left\{\eta_{n}\right\}_{n=0}^{\infty}$ and observable process $\left\{\xi_{n}\right\}_{n=0}^{\infty}$.

Definition 1 The process $\zeta=(\xi, \eta)$ is called an HTIMC if it follows the following form and conditions:

1. Suppose that a given time inhomogeneous Markov chain takes values in state space $\mathcal{Y}$, its starting distribution is

$$
\left(q\left(\omega_{0}\right), q\left(\omega_{1}\right) ; \ldots ; q\left(\omega_{b}\right)\right), \quad q\left(\omega_{i}\right)>0, \omega_{i} \in \mathcal{Y}
$$

and transition matrices are

$$
\mathcal{Q}_{k}=\left(q_{k}\left(\omega_{j} \mid \omega_{i}\right)\right), \quad q_{k}\left(\omega_{j} \mid \omega_{i}\right)>0, \omega_{i}, \omega_{j} \in \mathcal{Y}, k \geq 1
$$

where

$$
q_{k}\left(\omega_{j} \mid \omega_{i}\right)=\mathbb{P}\left(\eta_{k}=\omega_{j} \mid \eta_{k-1}=\omega_{i}\right), \quad k \geq 1
$$

2. For any positive integer $n$,

$$
\mathbb{P}\left(\xi_{0}=x_{0}, \ldots, \xi_{n}=x_{n} \mid \eta\right)=\prod_{k=0}^{n} \mathbb{P}\left(\xi_{k}=x_{k} \mid \eta_{k}\right) \quad \text { a.s. }
$$

Some necessary and sufficient conditions for (2.3) have been given by G.Q. Yang et al. [2].

(a) (2.3) holds if, for any $n$,

$$
\mathbb{P}\left(\xi_{0}=x_{0}, \ldots, \xi_{n}=x_{n} \mid \eta_{0}=y_{0}, \ldots, \eta_{n}=y_{n}\right)=\prod_{k=0}^{n} \mathbb{P}\left(\xi_{k}=x_{k} \mid \eta_{k}=y_{k}\right)
$$

holds.

(b) $\zeta=(\xi, \eta)$ is a hidden time inhomogeneous Markov chain if and only if $\forall n \geq 0$,

$$
p\left(x_{0}, y_{0}, \ldots, x_{n}, y_{n}\right)=q\left(y_{0}\right) \prod_{k=1}^{n} q_{k}\left(y_{k} \mid y_{k-1}\right) \prod_{k=0}^{n} p_{k}\left(x_{k} \mid y_{k}\right), \quad n \geq 1 .
$$

(c) $\zeta=(\xi, \eta)$ is a hidden time inhomogeneous Markov chain if and only if $\forall n \geq 0$,

$$
\begin{aligned}
& \mathbb{P}\left(\eta_{n}=y_{n} \mid \xi_{0}=x_{0}, \ldots, \xi_{n-1}=x_{n-1}, \eta_{0}=y_{0}, \ldots, \eta_{n-1}=y_{n-1}\right) \\
& \quad=\mathbb{P}\left(\eta_{n}=y_{n} \mid \eta_{n-1}=y_{n-1}\right)
\end{aligned}
$$




$$
\begin{aligned}
& \mathbb{P}\left(\xi_{n}=x_{n} \mid \xi_{0}=x_{0}, \ldots, \xi_{n}=x_{n}, \eta_{0}=y_{0}, \ldots, \eta_{n-1}=y_{n-1}\right) \\
& \quad=\mathbb{P}\left(\xi_{n}=x_{n} \mid \eta_{n}=y_{n}\right) .
\end{aligned}
$$

Let $\left\{a_{n}, b_{n}\right\}$ be two sequences of nonnegative integers with $b_{n}$ converging to infinity as $n \rightarrow \infty$. Let $\mathcal{S}_{a_{n}, b_{n}}\left(\theta_{i}, \omega_{j}\right), \mathcal{W}_{a_{n}, b_{n}}\left(\omega_{i}\right), \mathcal{T}_{a_{n}, b_{n}}\left(\theta_{i}\right), \theta_{i} \in \mathcal{X}, \omega_{j} \in \mathcal{Y}$ be the number of ordered couples $\left(\theta_{i}, \omega_{j}\right)$ in $\left(\xi_{a_{n}+1}, \eta_{a_{n}+1}\right),\left(\xi_{a_{n}+2}, \eta_{a_{n}+2}\right), \ldots,\left(\xi_{a_{n}+b_{n}}, \eta_{a_{n}+b_{n}}\right)$, with $\omega_{i}$ among $\eta_{a_{n}+1}, \eta_{a_{n}+2}, \ldots, \eta_{a_{n}+b_{n}}$ and $\theta_{i}$ among $\xi_{a_{n}+1}, \xi_{a_{n}+2}, \ldots, \xi_{a_{n}+b_{n}}$, respectively.

It is easy to verify that

$$
\begin{aligned}
& \mathcal{S}_{a_{n}, b_{n}}\left(\theta_{i}, \omega_{j}\right)=\sum_{k=a_{n}+1}^{a_{n}+b_{n}} 1_{\left\{\theta_{i}\right\}}\left(\xi_{k}\right) 1_{\left\{\omega_{j}\right\}}\left(\eta_{k}\right), \\
& \mathcal{W}_{a_{n}, b_{n}}\left(\omega_{i}\right)=\sum_{k=a_{n}+1}^{a_{n}+b_{n}} 1_{\left\{\omega_{i}\right\}}\left(\eta_{k}\right),
\end{aligned}
$$

and

$$
\mathcal{T}_{a_{n}, b_{n}}\left(\theta_{i}\right)=\sum_{k=a_{n}+1}^{a_{n}+b_{n}} 1_{\left\{\theta_{i}\right\}}\left(\xi_{k}\right)
$$

where $1_{A}(\cdot)$ denotes the indicator function of set $A$.

Lemma 1 Let $\zeta=(\xi, \eta)=\left\{\left(\xi_{k}, \eta_{k}\right)\right\}_{k=0}^{\infty}$ be an HTIMC which takes values in $\mathcal{X} \times \mathcal{Y}$, let $\left\{f_{k}(x, y)\right\}_{k=0}^{\infty}$ be a sequence of functions on $\mathcal{X} \times \mathcal{Y}$, let $\mathcal{F}_{m, n}=\sigma\left\{\left(\xi_{m}, \eta_{m}, \ldots, \xi_{n}, \eta_{n}\right), 0 \leq m \leq\right.$ $\left.n \in Z_{+}\right\}$, and let $\left\{a_{n}, b_{n}\right\}$ be a sequence of pairs of positive integers with $\sum_{n=1}^{\infty} \exp \left[-\varepsilon b_{n}\right]<\infty$, where $\varepsilon>0$ is arbitrary. Define

$$
\begin{aligned}
A(\alpha) & =\left\{\omega: \limsup _{n \rightarrow \infty} \frac{1}{b_{n}} \sum_{k=a_{n}+1}^{a_{n}+b_{n}} \mathbb{E}\left[f_{k}^{2}\left(\xi_{k}, \eta_{k}\right) e^{\alpha\left|f_{k}\left(\xi_{k}, \eta_{k}\right)\right|} \mid \mathcal{F}_{a_{n}, k-1}\right]=M(\alpha, \omega)<\infty\right\} \\
(\alpha>0) . &
\end{aligned}
$$

Then

$$
\lim _{n \rightarrow \infty} \frac{1}{b_{n}} \sum_{k=a_{n}+1}^{a_{n}+b_{n}}\left\{f_{k}\left(\xi_{k}, \eta_{k}\right)-\mathbb{E}\left[f_{k}\left(\xi_{k}, \eta_{k}\right) \mid \mathcal{F}_{a_{n}, k-1}\right]\right\}=0 \quad \text { a.s. } \omega \in A(\alpha)
$$

Proof Let $\lambda$ be a real number. We first define

$$
t_{a_{n}, b_{n}}(\lambda, \omega)=\frac{e^{\lambda \sum_{k=a_{n}+1}^{a_{n}+b_{n}} f_{k}\left(\xi_{k}, \eta_{k}\right)}}{\prod_{k=a_{n}+1}^{a_{n}+b_{n}} \mathbb{E}\left[e^{\lambda f_{k}\left(\xi_{k}, \eta_{k}\right)} \mid \mathcal{F}_{a_{n}, k-1}\right]} .
$$

Note that

$$
t_{a_{n}, b_{n}}(\lambda, \omega)=t_{a_{n}, b_{n-1}}(\lambda, \omega) \frac{e^{\lambda f_{a_{n}+b_{n}}\left(\xi_{a n+b n}, \eta_{a n+b n}\right)}}{\mathbb{E}\left[e^{\lambda f_{a_{n}+b_{n}}\left(\xi_{a n+b n}, \eta_{a n+b n}\right)} \mid \mathcal{F}_{a_{n}, a_{n}+b_{n}-1}\right]}
$$


and

$$
\mathbb{E}\left[t_{a_{n}, b_{n}}(\lambda, \omega)\right]=\mathbb{E}\left\{\mathbb{E}\left[t_{a_{n}, b_{n}}(\lambda, \omega)\right] \mid \mathcal{F}_{a_{n}, a_{n}+b_{n}-1}\right\}
$$

Hence, we have

$$
\mathbb{E}\left[t_{a_{n}, b_{n}}(\lambda, \omega) \mid \mathcal{F}_{a_{n}, a_{n}+b_{n}-1}\right]=t_{a_{n}, b_{n-1}}(\lambda, \omega) \quad \text { a.s. }
$$

It is easy to show that $\mathbb{E}\left[t_{a_{n}, b_{n}(\lambda, \omega)}\right]=1 ; \forall n \geq 1$. This and the Markov inequality imply that, for every $\varepsilon>0$,

$$
\mathbb{P}\left[\frac{1}{b_{n}} \log t_{a_{n}, b_{n}}(\lambda, \omega) \geq \varepsilon\right]=\mathbb{P}\left[t_{a_{n}, b_{n}}(\lambda, \omega) \geq \exp (n \varepsilon)\right] \leq 1 \cdot \exp \left(-\varepsilon b_{n}\right) .
$$

Hence

$$
\sum_{n=1}^{\infty} \mathbb{P}\left[\frac{1}{b_{n}} \log t_{a_{n}, b_{n}}(\lambda, \omega) \geq \varepsilon\right] \leq \sum_{n=1}^{\infty} \exp \left(-\varepsilon b_{n}\right)<\infty
$$

which, by the first Borel-Cantelli Lemma, allows us to conclude that $\lim \sup _{n} \frac{1}{b_{n}} \log t_{a_{n}, b_{n}}(s$, $\omega)<\varepsilon$ a.s., since $\varepsilon$ is arbitrary, thus

$$
\limsup _{n \rightarrow \infty} \frac{1}{b_{n}} \log t_{a_{n}, b_{n}}(\lambda, \omega) \leq 0 \quad \text { a.s. }
$$

follows since $\frac{1}{b_{n}} \log n^{2}=\frac{2 \log n}{b_{n}} \rightarrow 0(n \rightarrow \infty)$. We have by Eqs. (2.13) and (2.14) that

$$
\limsup _{n \rightarrow \infty} \frac{1}{b_{n}}\left\{\lambda \sum_{k=a_{n}+1}^{a_{n}+b_{n}} f_{k}\left(\xi_{k}, \eta_{k}\right)-\sum_{k=a_{n}+1}^{a_{n}+b_{n}} \log \mathbb{E}\left[e^{\lambda f_{k}\left(\xi_{k}, \eta_{k}\right)} \mid \mathcal{F}_{a_{n}, k-1}\right]\right\} \leq 0 \quad \text { a.s. }
$$

Taking $0<\lambda \leq \alpha$, and dividing both sides of Eq. (2.15) by $\lambda$, we get

$$
\limsup _{n \rightarrow \infty} \frac{1}{b_{n}}\left\{\sum_{k=a_{n}+1}^{a_{n}+b_{n}} f_{k}\left(\xi_{k}, \eta_{k}\right)-\sum_{k=a_{n}+1}^{a_{n}+b_{n}} \frac{\log \mathbb{E}\left[e^{\lambda f_{k}\left(\xi_{k}, \eta_{k}\right)} \mid \mathcal{F}_{a_{n}, k-1}\right]}{\lambda}\right\} \leq 0 \quad \text { a.s. }
$$

We have by Eq. (2.16) and inequalities $\log x \leq x-1(x>0), 0 \leq e^{x}-1-x \leq \frac{1}{2} x^{2} e^{|x|}$ that

$$
\begin{aligned}
& \limsup _{n \rightarrow \infty} \frac{1}{b_{n}} \sum_{k=a_{n}+1}^{a_{n}+b_{n}}\left\{f_{k}\left(\xi_{k}, \eta_{k}\right)-\mathbb{E}\left[f_{k}\left(\xi_{k}, \eta_{k}\right) \mid \mathcal{F}_{a_{n}, k-1}\right]\right\} \\
& \quad \leq \limsup _{n \rightarrow \infty} \frac{1}{b_{n}} \sum_{k=a_{n}+1}^{a_{n}+b_{n}}\left\{\frac{\log \mathbb{E}\left[e^{\lambda f_{k}\left(\xi_{k}, \eta_{k}\right)} \mid \mathcal{F}_{a_{n}, k-1}\right]}{\lambda}-\mathbb{E}\left[f_{k}\left(\xi_{k}, \eta_{k}\right) \mid \mathcal{F}_{a_{n}, k-1}\right]\right\} \\
& \quad \leq \limsup _{n \rightarrow \infty} \frac{1}{b_{n}} \sum_{k=a_{n}+1}^{a_{n}+b_{n}}\left\{\frac{\mathbb{E}\left[e^{\lambda f_{k}\left(\xi_{k}, \eta_{k}\right)} \mid \mathcal{F}_{a_{n}, k-1}\right]-1}{\lambda}-\mathbb{E}\left[f_{k}\left(\xi_{k}, \eta_{k}\right) \mid \mathcal{F}_{a_{n}, k-1}\right]\right\}
\end{aligned}
$$




$$
\begin{aligned}
& \leq \frac{\lambda}{2} \limsup _{n \rightarrow \infty} \frac{1}{b_{n}} \sum_{k=a_{n}+1}^{a_{n}+b_{n}} \mathbb{E}\left[f_{k}^{2}\left(\xi_{k}, \eta_{k}\right) e^{\alpha\left|f_{k}\left(\xi_{k}, \eta_{k}\right)\right|} \mid \mathcal{F}_{a_{n}, k-1}\right] \\
& =\frac{\lambda}{2} M(\alpha, \omega) \quad \text { a.s. } \omega \in A(\alpha) .
\end{aligned}
$$

Letting $\lambda \searrow 0^{+}$in Eq. (2.17), we get

$$
\limsup _{n \rightarrow \infty} \frac{1}{b_{n}} \sum_{k=a_{n}+1}^{a_{n}+b_{n}}\left\{f_{k}\left(\xi_{k}, \eta_{k}\right)-\mathbb{E}\left[f_{k}\left(\xi_{k}, \eta_{k}\right) \mid \mathcal{F}_{a_{n}, k-1}\right]\right\} \leq 0 \quad \text { a.s. } \omega \in A(\alpha) \text {. }
$$

Taking $-\alpha<\lambda \leq 0$, similarly, we have

$$
\begin{aligned}
& \lim _{n \rightarrow \infty} \frac{1}{b_{n}} \sum_{k=a_{n}+1}^{a_{n}+b_{n}}\left\{f_{k}\left(\xi_{k}, \eta_{k}\right)-\mathbb{E}\left[f_{k}\left(\xi_{k}, \eta_{k}\right) \mid \mathcal{F}_{a_{n}, k-1}\right]\right\} \\
& \geq \frac{\lambda}{2} M(\alpha, \omega) \quad \text { a.s. } \omega \in A(\alpha) .
\end{aligned}
$$

Putting $\lambda \nearrow 0^{-}$, we have

$$
\lim _{n \rightarrow \infty} \frac{1}{b_{n}} \sum_{k=a_{n}+1}^{a_{n}+b_{n}}\left\{f_{k}\left(\xi_{k}, \eta_{k}\right)-\mathbb{E}\left[f_{k}\left(\xi_{k}, \eta_{k}\right) \mid \mathcal{F}_{a_{n}, k-1}\right]\right\} \geq 0 \quad \text { a.s. } \omega \in A(\alpha) .
$$

From Eqs. (2.18) and (2.19), we obtain

$$
\lim _{n \rightarrow \infty} \frac{1}{b_{n}} \sum_{k=a_{n}+1}^{a_{n}+b_{n}}\left\{f_{k}\left(\xi_{k}, \eta_{k}\right)-\mathbb{E}\left[f_{k}\left(\xi_{k}, \eta_{k}\right) \mid \mathcal{F}_{a_{n}, k-1}\right]\right\}=0 \quad \text { a.s. } \omega \in A(\alpha)
$$

Thus we complete the proof of Lemma 1.

Lemma 2 Assume that $\zeta=(\xi, \eta)=\left\{\left(\xi_{k}, \eta_{k}\right)\right\}_{k=0}^{\infty}$ is an HTIMC defined as in Lemma 1. Then, for every $j<k ; k \geq 1$,

$$
\mathbb{E}\left[f_{k}\left(\xi_{k}, \eta_{k}\right) \mid \mathcal{F}_{j, k-1}\right]=\mathbb{E}\left[f_{k}\left(\xi_{k}, \eta_{k}\right) \mid \eta_{k-1}\right] \text { a.s. }
$$

Proof From definition of Hidden Markov chain, we have, for every $x_{i} \in \mathcal{X}, y_{j} \in \mathcal{Y}, m \leq n$; $n \geq 1$,

$$
\begin{aligned}
& \mathbb{p}\left(\xi_{n}=x_{n}, \eta_{n}=y_{n} \mid \xi_{m}=x_{m}, \eta_{m}=y_{m}, \ldots, \xi_{\mathrm{n}-1}=x_{\mathrm{n}-1}, \eta_{\mathrm{n}-1}=y_{\mathrm{n}-1}\right) \\
&= \mathbb{P}\left(\xi_{n}=x_{n} \mid \xi_{m}=x_{m}, \eta_{m}=y_{m}, \ldots, \xi_{\mathrm{n}-1}=x_{\mathrm{n}-1}, \eta_{\mathrm{n}-1}=y_{\mathrm{n}-1}, \eta_{n}=y_{n}\right) \\
& \quad \times \mathbb{p}\left(\eta_{n}=y_{n} \mid \xi_{m}=x_{m}, \eta_{m}=y_{m}, \ldots, \xi_{\mathrm{n}-1}=x_{\mathrm{n}-1}, \eta_{\mathrm{n}-1}=y_{\mathrm{n}-1}, \xi_{n}=x_{n}\right) \\
&= \mathbb{p}\left(\xi_{n}=x_{n} \mid \eta_{n}=y_{n}\right) \cdot \mathbb{p}\left(\eta_{n}=y_{n} \mid \eta_{\mathrm{n}-1}=y_{\mathrm{n}-1}, \xi_{n}=x_{n}\right) \\
&= \mathbb{P}\left(\xi_{n}=x_{n}, \eta_{n}=y_{n} \mid \eta_{\mathrm{n}-1}=y_{\mathrm{n}-1}\right) .
\end{aligned}
$$

Hence, we have

$$
\mathbb{E}\left[f_{k}\left(\xi_{k}, \eta_{k}\right) \mid \mathcal{F}_{j, k-1}\right]
$$




$$
\begin{aligned}
& =\sum_{x_{k} \in \mathcal{X}} \sum_{y_{k} \in \mathcal{Y}} f_{k}\left(x_{k}, y_{k}\right) \mathbb{P}\left(\xi_{k}=x_{k}, \eta_{k}=y_{k} \mid \xi_{j}=x_{j}, \eta_{j}=y_{j}, \ldots, \xi_{k-1}=x_{k-1}, \eta_{k-1}=y_{k-1}\right) \\
& =\sum_{x_{k} \in \mathcal{X}} \sum_{y_{k} \in \mathcal{Y}} f_{k}\left(x_{k}, y_{k}\right) \mathbb{P}\left(\xi_{k}=x_{k}, \eta_{k}=y_{k} \mid \eta_{k-1}=y_{k-1}\right) \\
& =\mathbb{E}\left[f_{k}\left(\xi_{k}, \eta_{k}\right) \mid \eta_{k-1}\right] .
\end{aligned}
$$

According to Theorem 1 of Wang [5], it is easy to verify the following lemma.

Lemma 3 Suppose that $\eta=\left(\eta_{0}, \eta_{1}, \ldots\right)$ is a time inhomogeneous Markov chain which takes value in state space $\mathcal{Y}$, its starting distribution is

$$
\left(q\left(\omega_{0}\right), q\left(\omega_{1}\right) ; \ldots ; q\left(\omega_{b}\right)\right), \quad q\left(\omega_{i}\right)>0, \omega_{i} \in \mathcal{Y}
$$

and transition matrices are

$$
\mathcal{Q}_{k}=\left(q_{k}\left(\omega_{j} \mid \omega_{i}\right)\right), \quad q_{k}\left(\omega_{j} \mid \omega_{i}\right)>0, \omega_{i}, \omega_{j} \in \mathcal{Y}, k \geq 1
$$

where

$$
q_{k}\left(\omega_{j} \mid \omega_{i}\right)=\mathbb{P}\left(\eta_{k}=\omega_{j} \mid \eta_{k-1}=\omega_{i}\right), \quad k \geq 1
$$

Assume that $\Pi=\left(q\left(\omega_{i}, \omega_{j}\right)\right), q\left(\omega_{i}, \omega_{j}\right)>0, \omega_{i}, \omega_{j} \in \mathcal{Y}$ is another transition matrix which satisfies the following condition:

$$
\lim _{n \rightarrow \infty} \frac{1}{b_{n}} \sum_{k=a_{n}+1}^{a_{n}+b_{n}}\left|q_{k}\left(\omega_{i}, \omega_{j}\right)-q\left(\omega_{i}, \omega_{j}\right)\right|=0 \quad \forall \omega_{i}, \omega_{j} \in \mathcal{Y} .
$$

Then, for each $\omega_{s} \in \mathcal{Y}$,

$$
\lim _{n \rightarrow \infty} \frac{1}{b_{n}} \sum_{k=a_{n}+1}^{a_{n}+b_{n}} 1_{\left\{\omega_{s}\right\}}\left(\eta_{k-1}\right)=\pi_{s} \quad \text { a.s. }
$$

where $\left(\pi_{0}, \pi_{1}, \pi_{2}, \ldots, \pi_{b}\right)$ is the stationary distribution determined by $\Pi$.

\section{Main results}

Theorem 1 Let $\zeta=(\xi, \eta)=\left\{\left(\xi_{k}, \eta_{k}\right)\right\}_{k=0}^{\infty}$ be an HTIMC which takes values in $\mathcal{X} \times \mathcal{Y}, f(x, y)$ be a function on $\mathcal{X} \times \mathcal{Y}$.

Let $\Pi=\left(q\left(\omega_{i}, \omega_{j}\right)\right), q\left(\omega_{i}, \omega_{j}\right)>0, \omega_{i}, \omega_{j} \in \mathcal{Y}$ be another transition matrix and $p\left(\theta_{i} \mid\right.$ $\left.\omega_{j}\right),\left(\theta_{i}, \omega_{j}\right) \in \mathcal{X} \times \mathcal{Y}$ be conditional probabilities which satisfy

$$
\begin{array}{ll}
\lim _{n \rightarrow \infty} \frac{1}{b_{n}} \sum_{k=a_{n}+1}^{a_{n}+b_{n}}\left|q_{k}\left(\omega_{i}, \omega_{j}\right)-q\left(\omega_{i}, \omega_{j}\right)\right|=0 & \forall \omega_{i}, \omega_{j} \in \mathcal{Y}, \\
\lim _{n \rightarrow \infty} \frac{1}{b_{n}} \sum_{k=a_{n}+1}^{a_{n}+b_{n}}\left|p_{k}\left(\theta_{i} \mid \omega_{j}\right)-p\left(\theta_{i} \mid \omega_{j}\right)\right|=0 & \forall\left(\theta_{i}, \omega_{j}\right) \in \mathcal{X} \times \mathcal{Y} .
\end{array}
$$


If the transition matrix $\Pi$ has a stationary distribution $\pi=\left(\pi_{0}, \pi_{1}, \pi_{2}, \ldots, \pi_{b}\right)$, then

$$
\lim _{n \rightarrow \infty} \frac{1}{b_{n}} \sum_{k=a_{n}+1}^{a_{n}+b_{n}} f\left(\xi_{k}, \eta_{k}\right)=\sum_{\theta_{i} \in \mathcal{X}} \sum_{\omega_{j} \in \mathcal{Y}} \sum_{\omega_{s} \in \mathcal{Y}} \pi_{s} f\left(\theta_{i}, \omega_{j}\right) q\left(\omega_{s}, \omega_{j}\right) p\left(\theta_{i} \mid \omega_{s}\right) \quad \text { a.s. }
$$

Proof Since $f(x, y)$ is bounded, we have by Lemmas 1 and 2 that

$$
\lim _{n \rightarrow \infty} \frac{1}{b_{n}} \sum_{k=a_{n}+1}^{a_{n}+b_{n}}\left\{f\left(\xi_{k}, \eta_{k}\right)-\mathbb{E}\left[f\left(\xi_{k}, \eta_{k}\right) \mid \eta_{k-1}\right]\right\}=0 \quad \text { a.s. }
$$

Observe that

$$
\mathbb{E}\left[f\left(\xi_{k}, \eta_{k}\right) \mid \eta_{k-1}\right]=\sum_{\theta_{i} \in \mathcal{X}} \sum_{\omega_{j} \in \mathcal{Y}} f\left(\theta_{i}, \omega_{j}\right) q_{k}\left(\eta_{k-1}, \omega_{j}\right) p_{k}\left(\theta_{i} \mid \eta_{k-1}\right) .
$$

We have that, by Eq. (3.4),

$$
\begin{aligned}
& \lim \sup _{n \rightarrow \infty}\left|\frac{1}{b_{n}} \sum_{k=a_{n}+1}^{a_{n}+b_{n}} f\left(\xi_{k}, \eta_{k}\right)-\sum_{\theta_{i} \in \mathcal{X}} \sum_{\omega_{j} \in \mathcal{Y}} \sum_{\omega_{s} \in \mathcal{Y}} \pi_{s} f\left(\theta_{i}, \omega_{j}\right) q\left(\omega_{s}, \omega_{j}\right) p\left(\theta_{i} \mid \omega_{s}\right)\right| \\
& \leq \lim \sup _{n \rightarrow \infty}\left|\frac{1}{b_{n}} \sum_{k=a_{n}+1}^{a_{n}+b_{n}} \mathbb{E}\left[f\left(\xi_{k}, \eta_{k}\right) \mid \eta_{k-1}\right]-\sum_{\theta_{i} \in \mathcal{X}} \sum_{\omega_{j} \in \mathcal{Y}} \sum_{\omega_{s} \in \mathcal{Y}} \pi_{s} f\left(\theta_{i}, \omega_{j}\right) q\left(\omega_{s}, \omega_{j}\right) p\left(\theta_{i} \mid \omega_{s}\right)\right| \\
& =\lim \sup _{n \rightarrow \infty} \mid \frac{1}{b_{n}} \sum_{k=a_{n}+1}^{a_{n}+b_{n}} \sum_{\theta_{i} \in \mathcal{X}} \sum_{\omega_{j} \in \mathcal{Y}} f\left(\theta_{i}, \omega_{j}\right) q_{k}\left(\eta_{k-1}, \omega_{j}\right) p_{k}\left(\theta_{i} \mid \eta_{k-1}\right) \\
& -\sum_{\theta_{i} \in \mathcal{X}} \sum_{\omega_{j} \in \mathcal{Y}} \sum_{\omega_{s} \in \mathcal{Y}} \pi_{s} f\left(\theta_{i}, \omega_{j}\right) q\left(\omega_{s}, \omega_{j}\right) p\left(\theta_{i} \mid \omega_{s}\right) \\
& =\lim \sup _{n \rightarrow \infty} \mid \frac{1}{b_{n}} \sum_{k=a_{n}+1}^{a_{n}+b_{n}} \sum_{\theta_{i} \in \mathcal{X}} \sum_{\omega_{j} \in \mathcal{Y}} \sum_{\omega_{s} \in \mathcal{Y}} 1_{\left\{\omega_{s}\right\}}\left(\eta_{k-1}\right) \pi_{s} f\left(\theta_{i}, \omega_{j}\right) q_{k}\left(\omega_{s}, \omega_{j}\right) p_{k}\left(\theta_{i} \mid \omega_{s}\right) \\
& -\sum_{\theta_{i} \in \mathcal{X}} \sum_{\omega_{j} \in \mathcal{Y}} \sum_{\omega_{s} \in \mathcal{Y}} \pi_{s} f\left(\theta_{i}, \omega_{j}\right) q\left(\omega_{s}, \omega_{j}\right) p\left(\theta_{i} \mid \omega_{s}\right) \\
& =\lim \sup _{n \rightarrow \infty} \mid \frac{1}{b_{n}} \sum_{k=a_{n}+1}^{a_{n}+b_{n}} \sum_{\theta_{i} \in \mathcal{X}} \sum_{\omega_{j} \in \mathcal{Y}} \sum_{\omega_{s} \in \mathcal{Y}} 1_{\left\{\omega_{s}\right\}}\left(\eta_{k-1}\right) f\left(\theta_{i}, \omega_{j}\right) \\
& \times\left[\left(q_{k}\left(\omega_{s}, \omega_{j}\right)-q\left(\omega_{s}, \omega_{j}\right)\right) p_{k}\left(\theta_{i} \mid \omega_{s}\right)\right. \\
& \left.+q\left(\omega_{s}, \omega_{j}\right)\left(p_{k}\left(\theta_{i} \mid \omega_{s}\right)-p\left(\theta_{i} \mid \omega_{s}\right)\right)+p\left(\theta_{i} \mid \omega_{s}\right) q\left(\omega_{s}, \omega_{j}\right)\right] \\
& -\sum_{\theta_{i} \in \mathcal{X}} \sum_{\omega_{j} \in \mathcal{Y}} \sum_{\omega_{s} \in \mathcal{Y}} \pi_{s} f\left(\theta_{i}, \omega_{j}\right) q\left(\omega_{s}, \omega_{j}\right) p\left(\theta_{i} \mid \omega_{s}\right) \\
& \leq \lim \sup _{n \rightarrow \infty} \sum_{\theta_{i} \in \mathcal{X}} \sum_{\omega_{j} \in \mathcal{Y}} \sum_{\omega_{s} \in \mathcal{Y}} \sup _{\theta_{i} \in \mathcal{X}, \omega_{j} \in \mathcal{Y}}\left|f\left(\theta_{i}, \omega_{j}\right)\right|\left\{\left|\frac{1}{b_{n}} \sum_{k=a_{n}+1}^{a_{n}+b_{n}}\right| q_{k}\left(\omega_{s}, \omega_{j}\right)-q\left(\omega_{s}, \omega_{j}\right) \mid\right.
\end{aligned}
$$




$$
\left.+\frac{1}{b_{n}} \sum_{k=a_{n}}^{a_{n}+b_{n}}\left|p_{k}\left(\theta_{i} \mid \omega_{s}\right)-p\left(\theta_{i} \mid \omega_{s}\right)\right| \mid\right\}
$$

$=0$.

Therefore Eq. (3.3) holds.

Corollary 1 Under the conditions of Theorem 1 , we have for each $\theta_{i^{\prime}} \in \mathcal{X}, \omega_{j^{\prime}}, \omega_{s} \in \mathcal{Y}$,

$$
\lim _{n \rightarrow \infty} \frac{1}{b_{n}} \mathcal{S}_{a_{n}, b_{n}}\left(\theta_{i^{\prime}}, \omega_{j^{\prime}}\right)=\sum_{\omega_{s} \in \mathcal{Y}} \pi_{s} q\left(\omega_{s}, \omega_{j^{\prime}}\right) p\left(\theta_{i^{\prime}} \mid \omega_{s}\right) \quad \text { a.s. }
$$

Proof Put $f(x, y)=1_{\left\{\theta_{i^{\prime}}\right\}}(x) 1_{\left\{\omega_{j^{\prime}}\right\}}(y),\left(\theta_{i^{\prime}}, \omega_{j^{\prime}}\right) \in \mathcal{X} \times \mathcal{Y}$ in Theorem 1 . Then

$$
\begin{aligned}
\lim _{n \rightarrow \infty} & \frac{1}{b_{n}} \sum_{k=a_{n}+1}^{a_{n}+b_{n}} f\left(\xi_{k}, \eta_{k}\right)-\sum_{\theta_{i} \in \mathcal{X}} \sum_{\omega_{j} \in \mathcal{Y}} \sum_{\omega_{s} \in \mathcal{Y}} \pi_{s} f\left(\theta_{i}, \omega_{j}\right) q\left(\omega_{s}, \omega_{j}\right) p\left(\theta_{i} \mid \omega_{s}\right) \\
= & \lim _{n \rightarrow \infty} \frac{1}{b_{n}} \sum_{k=a_{n}+1}^{a_{n}+b_{n}} 1_{\left\{\theta_{i^{\prime}}\right\}}\left(\xi_{k}\right) 1_{\left\{\omega_{j^{\prime}}\right\}}\left(\eta_{k}\right) \\
& -\sum_{\theta_{i} \in \mathcal{X}} \sum_{\omega_{j} \in \mathcal{Y}} \sum_{\omega_{s} \in \mathcal{Y}} 1_{\left\{\theta_{i^{\prime}}\right\}}\left(\theta_{i}\right) 1_{\left\{\omega_{j^{\prime}}\right\}}\left(\omega_{j}\right) \pi_{s} q\left(\omega_{s}, \omega_{j}\right) p\left(\theta_{i} \mid \omega_{s}\right) \\
= & \lim _{n \rightarrow \infty} \frac{1}{b_{n}} \mathcal{S}_{a_{n}, b_{n}}\left(\theta_{i^{\prime}}, \omega_{j^{\prime}}\right)-\sum_{\omega_{s} \in \mathcal{Y}} \pi_{s} q\left(\omega_{s}, \omega_{j^{\prime}}\right) p\left(\theta_{i^{\prime}} \mid \omega_{s}\right)=0 \quad \text { a.s. }
\end{aligned}
$$

Corollary 2 Under the assumptions of Theorem 1 , we have, for each $\theta_{i^{\prime \prime}} \in \mathcal{X}, \omega_{s} \in \mathcal{Y}$,

$$
\lim _{n \rightarrow \infty} \frac{1}{b_{n}} \mathcal{T}_{a_{n}, b_{n}}\left(\theta_{i^{\prime \prime}}\right)=\sum_{\omega_{s} \in \mathcal{Y}} \pi_{s} p\left(\theta_{i^{\prime \prime}} \mid \omega_{s}\right) \quad \text { a.s. }
$$

Proof Put $f(x, y)=1_{\left\{\theta_{i^{\prime \prime}}\right\}}(x),(x, y) \in \mathcal{X} \times \mathcal{Y}$ in Theorem 1. Then

$$
\begin{aligned}
& \lim _{n \rightarrow \infty} \frac{1}{b_{n}} \sum_{k=a_{n}+1}^{a_{n}+b_{n}} f\left(\xi_{k}, \eta_{k}\right)-\sum_{\theta_{i} \in \mathcal{X}} \sum_{\omega_{j} \in \mathcal{Y}} \sum_{\omega_{s} \in \mathcal{Y}} \pi_{s} f\left(\theta_{i}, \omega_{j}\right) q\left(\omega_{s}, \omega_{j}\right) p\left(\theta_{i} \mid \omega_{s}\right) \\
& =\lim _{n \rightarrow \infty} \frac{1}{b_{n}} \sum_{k=a_{n}+1}^{a_{n}+b_{n}} 1_{\left\{\theta_{i^{\prime \prime}}\right\}}\left(\xi_{k}\right)-\sum_{\theta_{i} \in \mathcal{X}} \sum_{\omega_{j} \in \mathcal{Y}} \sum_{\omega_{s} \in \mathcal{Y}} 1_{\left\{\theta_{i^{\prime \prime}}\right\}}\left(\theta_{i}\right) \pi_{s} q\left(\omega_{s}, \omega_{j}\right) p\left(\theta_{i} \mid \omega_{s}\right) \\
& =\lim _{n \rightarrow \infty} \frac{1}{b_{n}} \mathcal{T}_{a_{n}, b_{n}}\left(\theta_{i^{\prime \prime}}\right)-\sum_{\omega_{s} \in \mathcal{Y}} \pi_{s} p\left(\theta_{i^{\prime \prime}} \mid \omega_{s}\right)=0 \quad \text { a.s. }
\end{aligned}
$$

This research is supported in part by the RP of AnHui Provincial Department of Education (KJ2017A851, KJ2017A547).

\section{Competing interests}

The authors declare that they have no competing interests.

\section{Authors' contributions}

All authors carried out the proof. All authors conceived of the study, and participated in its design and coordination. All authors read and approved the final manuscript. 


\section{Author details}

'School of Mathematics, Maanshan Teachers' College, Maanshan, China. ${ }^{2}$ Department of Mathematics and Physics, HeFei University, Anhui, P.R. China. ${ }^{3}$ School of Mathematics \& Physics, AnHui University of Technology, Ma'anshan, China.

\section{Publisher's Note}

Springer Nature remains neutral with regard to jurisdictional claims in published maps and institutional affiliations.

Received: 1 March 2018 Accepted: 17 October 2018 Published online: 25 October 2018

\section{References}

1. Baum, L.E., Petrie, T.: Statistical inference for probabilistic functions of finite state Markov chains. Ann. Math. Stat. 37, 1554-1563 (1966)

2. Yang, G.Q., Yang, W.G., Wu, X.T.: The strong laws of large numbers for countable nonhomogeneous hidden Markov models. Commun. Stat., Theory Methods 46(17), 8808-8819 (2017)

3. Zygmund, A.: Trigonometric Series, vol. 1. Cambridge University Press, New York (1959)

4. Gut, A., Stradtmüller, U.: On the strong law of large numbers for delayed sums and random fields. Acta Math. Hung. 129(1-2), 182-203 (2010)

5. Wang, Z.Z., Yang, W.G.: The generalized entropy ergodic theorem for nonhomogeneous Markov chains. J. Theor. Probab. 29, 761-775 (2016)

6. Wang, Z.Z.: A kind of asymptotic properties of moving averages for Markov chains in Markovian environments. Commun. Stat., Theory Methods 46(22), 10926-10942 (2017)

7. Wang, Z.Z:: Some limit theorems of delayed sums for row-wise conditionally independent stochastic arrays. Commun. Stat., Theory Methods 46(11), 5265-5272 (2017)

8. Isaacson, D., Madsen, R.: Markov Chains Theory and Applications. Wiley, New York (1976)

\section{Submit your manuscript to a SpringerOpen ${ }^{\odot}$ journal and benefit from:}

- Convenient online submission

- Rigorous peer review

- Open access: articles freely available online

- High visibility within the field

- Retaining the copyright to your article

Submit your next manuscript at $\boldsymbol{~ s p r i n g e r o p e n . c o m ~}$ 